\title{
Assessment of Patients with Hirschsprung Disease and the Use of Laparoscopy
}

\author{
Mesut Demir, (1) Melih Akin, (1) Meltem Kaba, (1) Nimetullah Mete Genc, (1) Nihat Sever, \\ (D) Cetin Ali Karadag, (D) Ali Ihsan Dokucu
}

Department of Pediatrics, Sisli Hamidiye Etfal Training and Research Hospital, Istanbul, Turkey

\begin{abstract}
Objectives: This study aims to evaluate patients who were diagnosed and treated due to Hirschsprung disease (HD) in our clinic. Methods: We retrospectively evaluated the demographic and clinical findings of the patients with HD, who were operated in our clinic between January 2010 and December 2015.

Results: During study period, 28 patients (19 male 9 female) were found to be operated due to HD in our clinic. Mean age was 16.8 months (1-168). "Transanal Endorectal Pull-through (TERPT)" was performed to 20 of them, Duhamell procedure to five and Soave procedure to three of them. TERPT was applied as laparoscopy assisted in four of them and biopsies were taken laparoscopically preoperatively from one of the patients from each group. Soave procedure was performed in three patients; one had anal stenosis and history of recurrent enterocolitis after TERPT procedure and pathologic analysis revealed neuronal intestinal dysplasia and the other one had total colonic HD and performed Soave procedure with colonic patch. Seven (25\%) patients had enterocolitis. Frequencies of enterocolitis were three in two patients, two in two patients and one in three patients. Broad spectrum anibiotics and rectal washouts were supplied to these patients. Five of the seven patients with enterocolitis were operated with TERPT; two patients were operated with Duhamell procedure. Only one of them had total colonic HD. Three patients had total colonic HD diagnosis. Two of them were operated with Duhamell-Martin procedure and one was with Soave procedure with colonic patch according to Kimura technique. Anal stenosis developed in two patients after TERPT and treated with dilatations. Soiling rate was $3 \%(1 / 28)$ and this single patient was treated with laxatives and toilet training. Mean duration of hospitalization was 8.75 (2-14) days. Mean length of the removed intestinal segment was $23.6(5-38) \mathrm{cm}$. Mean follow-up was for 35.5 (2-56) months. Neither of the patients was followed in the intensive care unit postoperatively nor died.

Conclusion: TERPT procedure win priority in HD, but other procedures keep importance. Recently, laparoscopy-assisted TERPT is preferred in our clinic in HD therapy due to easy biopsy, full exposure to the transitional zone, the advantage of meso preparation of colon and prevention of strained anastomosis.
\end{abstract}

Keywords: Enterocolitis; hirschsprung disease; laparoscopy.

Please cite this article as "Demir M, Akin M, Kaba M, Genc NM, Sever N, Karadag CA, et al. Assessment of Patients with Hirschsprung Disease and the Use of Laparoscopy. Med Bull Sisli Etfal Hosp 2020;54(2):218-221".

$\mathrm{H}^{\prime}$ irschsprung Disease (HD) is a congenital disease seen at $1 / 5000$ births. HD is more common in men. ${ }^{[1,2]} \mathrm{HD}$ is a disease characterized by the absence of ganglion cells in the myenteric and submucosal plexuses of the distal intestines. Today, single-stage surgery is the standard treatment, and the laparoscopic approach is now widely used to guide this procedure. The principles of the operation include removal of the aganglionic colon and anastomosis of the normally innervated intestine immediately proximal to the anus. ${ }^{[3]}$ In developing countries, $90 \%$ of the patients with $\mathrm{HH}$ are diagnosed within one year after birth. ${ }^{[4,5]}$ The complication rates are higher in patients diagnosed at a later period. ${ }^{[6]}$ 
Complaints begin with the delay of meconium transition at birth. If in $99 \%$ of newborns born at term, the passage of meconium does not occur within the first 48 hours HD should be considered. In some infants, the passage of meconium is normal. The disease manifests itself in the form of chronic constipation. As a result of lack of meconium passage, obstruction and ultimately distension in the abdomen develops. The anal tone is normal as palpated during the digital rectal examination (DRM). After DRM, there is an explosive and abundant foul-smelling stool and gas discharge.

The present study aims to evaluate the results of our patients who were diagnosed and treated for HD in our clinic.

\section{Methods}

In this study, the demographic and clinical findings of our patients who underwent surgical treatment for HD between January 2010 and December 2015 in our clinic were evaluated retrospectively concerning their ages at the time of surgery, length of hospital stay, and postoperative complications.

The diagnosis of HD was made by rectal biopsies performed following a physical examination, and colon x-ray. Laparoscopy for our patients was used to identify the aganglionic segment, to take serial biopsies to determine the level, and to prepare the vessels of the ganglionic segment to be lowered in order to assist in definitive surgery. The surgical treatment procedure was decided according to the surgeon's experience, stoma status of the patient, the treatment response of enterocolitis in the neonatal period and the length of the aganglionic segment.

\section{Results}

A total of 28 patients (19 M, 9F) who were followed up in our clinic with the diagnosis HD received treatment accordingly. The average age of the patients was 16.8 (1-168 months) months. A definitive diagnosis of patients was made with rectal biopsy performed before or during the operation.

Transanal Endorectal Pull-Through (TERPT) (Fig. 1) procedure was performed in 20 of 28 patients, Duhamell Procedure in five and Soave Procedure in three patients. In four of the 20 patients TERPT procedures were performed with the help of laparoscopy. Peroperative laparoscopy was performed in one of the remaining 16 patients and in one of five patients who underwent the Duhamel procedure and then serial biopsies were obtained (Fig. 2).

Soave procedure was applied to three patients. One of these patients underwent laparoscopy and preoperatively, biopsies were obtained (Table 1). One patient neuronal intestinal dysplasia diagnosed based on histopathological analysis

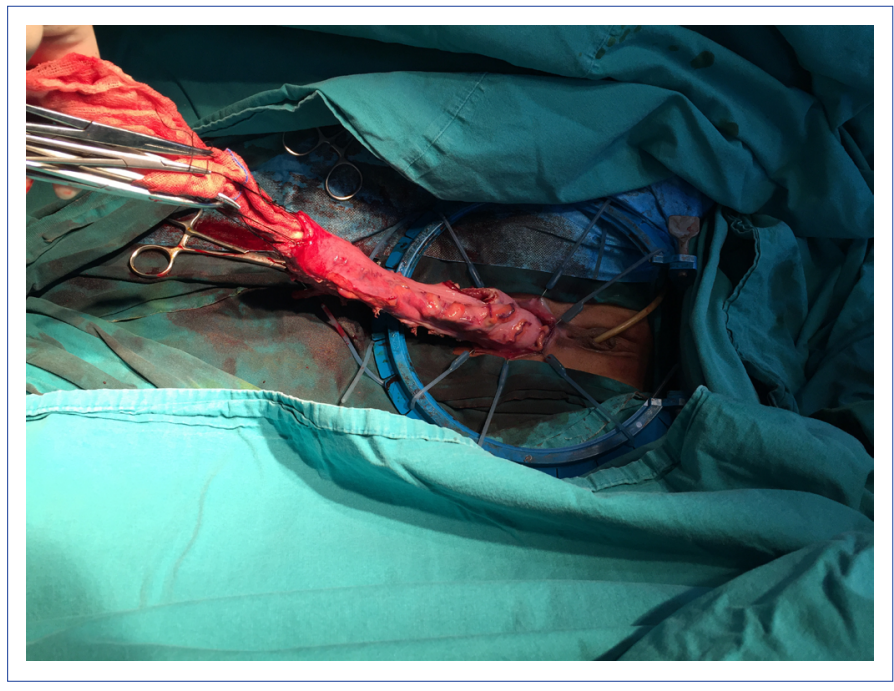

Figure 1. Transanal Endorectal Pull -through.

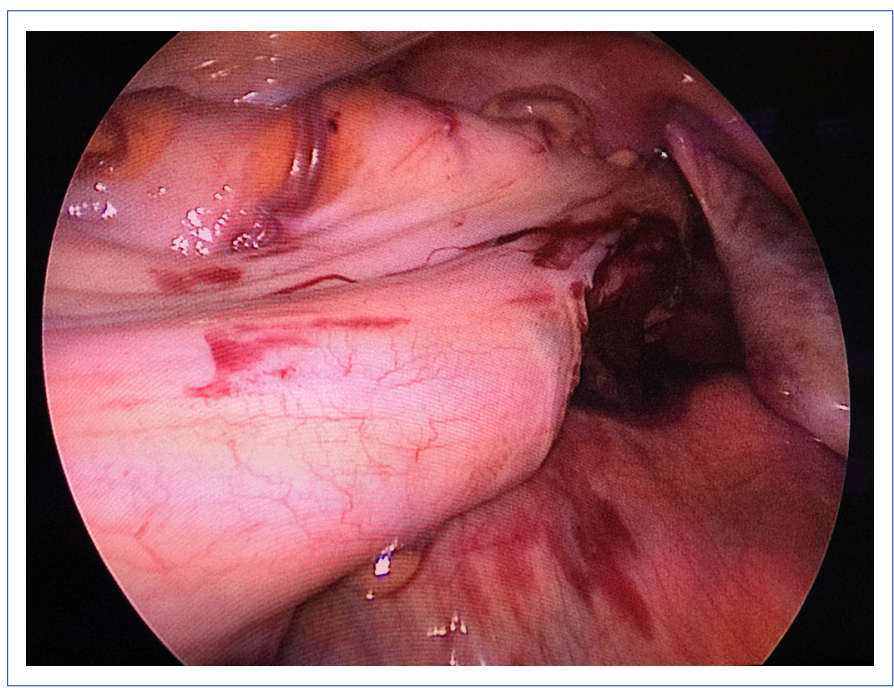

Figure 2. Laparoscopy-assisted TERPT procedure.

developed enterocolitis and stenosis after TERPT. The third patient who had total colonic HD underwent a colonic patch application. None of our patients required postoperative intensive care or exited. Average hospital stay was 8.75 (214 days) days. According to the results obtained from the pathology reports, the length of the intestinal segment removed was $23.6(5-38 \mathrm{~cm}) \mathrm{cm}$ (Table 2). Enterocolitis developed in $25 \%$ of our patients. While five (25\%) of our seven

Table 1. Surgical procedures applied for patients

\begin{tabular}{lccc}
\hline & TERPT & $\begin{array}{c}\text { Duhamel } \\
\text { Procedure }\end{array}$ & $\begin{array}{c}\text { Soave } \\
\text { Procedure }\end{array}$ \\
\hline Laparoscopic assisted & 20 & 5 & 3 \\
Preoperative laparoscopic biopsy & 1 & 1 & - \\
\hline
\end{tabular}


Table 2. Postoperative data of the patients

Average hospital stay (days)

8.75 (2-14) days

Length of bowel segment excised $(\mathrm{cm})$

$23.6(5-38) \mathrm{cm}$

Average follow-up period (month)

35.5 (2-56) months

Table 3. Postoperative complications of procedures applied

\begin{tabular}{lccc}
\hline & TERPT & Duhamell & Soave \\
\hline Enterocolitis & 5 & 2 & - \\
Stenosis & 2 & - & - \\
Anal incontinence & - & 1 & - \\
\hline
\end{tabular}

patients who developed enterocolitis had undergone TERPT and two (40\%) Duhamell operations. Only one of these patients had total colonic HD. Soave procedure was applied to two of total colonic HD patients using Duhamell-Martin procedure and colonic patch prepared with Kimura methodwas applied to one of these patients. Enterocolitis developed three times in two, twice in two and once in three patients during the follow-up period. These patients were treated with rectal wash-outs and broad-spectrum antibiotics. Anal stenosis developed in two patients who underwent TERPT and responded to serial rectal dilatations. Fecal incontinence was seen in $3 \%(1 / 28)$ of the patients who were treated with laxatives and toilet training (Table 3). The average follow-up period of the patients was 35.5 (2-56 months) months.

\section{Discussion}

Hirschsprung disease is a congenital disease mostly seen in men, characterized by the absence of ganglion of the distal intestinal segment. ${ }^{[7]} A$ variety of surgical methods have been described for its treatment. Among these methods, the TERPT procedure is accepted as a more popular method. ${ }^{[8,9]}$ All of the surgical procedures applied have their advantages and disadvantages. In our clinic we prefer the TERPT procedure as a priority for eligible patients. In addition, with the development of minimally invasive surgery in recent years, laparoscopy- assisted TERPT has become preferred by our clinic. Minimally invasive surgical treatment is an effective method for showing the aganglionic segment in HD, preventing exploration with wide incisions, and for vascular preparation during definitive surgery. In particular, the length of the aganglionic segment in patients undergoing TERPT can be estimated by preoperative colonic radiographs.

This condition causes serious problems during the operation in patients who undergo direct TERPT if the patient has long-segment HD. Because, in patients in whom the TERPT procedure is initiated, the possibility of performing the Duhamell or Duhamell-Martin Procedures is eliminated. Therefore, the laparoscopic approach will help to plan the treatment of long-segment HD patients by helping to diagnose long-segment HD in addition to biopsy and vascular preparation. At the same time, laparoscopic vascular preparation will reduce the complications that may occur due to tense anastomosis. In this way, the probability of anal stenosis that may occur postoperatively decreases. In addition, minimally invasive surgery is known to reduce the incidence of intraabdominal adhesions. ${ }^{[10]}$ Therefore, none of our patients undergoing TERPT and laparoscopic TERPT developed postoperative adhesion and the esthetic appearance was acceptable.

Long-term problems after pull-through are ongoing obstructive symptoms, soiling and enterocolitis. ${ }^{[11]}$ The frequency of enterocolitis in our series was found to be $25 \%$. However, when we scan the literature, we see that the frequency of enterecholite is between $4.4 \%$ and $14.2 \% .{ }^{[10]}$ In our series, we think that the reason why the frequency of enterocolitis is so frequent is the immature immune system. ${ }^{[12]}$ These patients also responded to medical treatment with rectal wash-out and broad-spectrum antibiotics. All children should be followed up regularly until at least five years of age. ${ }^{[3]}$ If these children have ongoing problems, they should be followed up longer. In our series, our average follow-up period was 35.5 (2-56 months) months. In long-term follow-up, an attack of enterocolitis, obstructive symptoms (30\%) and abdominal distension, vomiting or severe constipation, and associated anal incontinence may develop. ${ }^{[3]}$ Enterocolitis (25\%) and anal incontinence (3\%) were observed in our series.

\section{Conclusion}

In conclusion, the TERPT method has gained priority in the surgical treatment of $\mathrm{HD}$, and other surgical procedures also have retained their importance. Laparoscopy-assisted TERPT has taken its place in the treatment in our clinic because of its ease in taking a biopsy, its usefulness in the visualization of the transition zone, its advantage in preparing the vessels and making an unstretched anastomosis. Long-term problems after pull-through are obstructive symptoms, soiling and enterocolitis. Patients need longterm follow-up for these symptoms.

\section{Disclosures}

Ethics Committee Approval: Retrospective study.

Peer-review: Externally peer-reviewed.

Conflict of Interest: None declared.

Authorship Contributions: Concept - M.K.; Design - M.D., M.A.; Supervision - M.D.; Materials - M.A., M.D.; Data collection \&/or processing - Ç.A.K.; Analysis and/or interpretation - N.M.G., N.S.; Literature search - M.D., N.M.G.; Writing - M.D., M.A.; Critical review - A.I.D. 


\section{References}

1. Guerra J, Wayne C, Musambe T, Nasr A. Laparoscopic-assisted transanal pull-through (LATP) versus complete transanal pullthrough (CTP) in the surgical management of Hirschsprung's disease. J Pediatr Surg 2016;51:770-4. [CrossRef]

2. Nurko S. Hirschsprung's disease. American Motility Society (AMS) and the International Foundation for Functional Gastrointestinal Disorders (IFFGD). Available at: https://pdfs.semanticscholar.org/ 8ca2/24c0be767c7d2143d74888a118a706dca819.pdf. Accessed Apr 03, 2020.

3. Langer JC. Laparoscopic and transanal pull-through for Hirschsprung disease. Semin Pediatr Surg 2012;21:283-90. [CrossRef]

4. Sharma S, Gupta DK. Hirschsprung's disease presenting beyond infancy: surgical options and postoperative outcome. Pediatr Surg Int 2012;28:5-8. [CrossRef]

5. Ekenze SO, Ngaikedi C, Obasi AA. Problems and outcome of Hirschsprung's disease presenting after 1 year of age in a developing country. World J Surg 2011;35:22-6. [CrossRef]

6. Nam SH, Cho MJ, Kim DY. One-stage laparoscopy-assisted endorectal pull-through for late presented Hirschsprung's diseaseCase series. Int J Surg Case Rep 2015;16:162-5. [CrossRef]
7. Guerra J, Wayne C, Musambe T, Nasr A. Laparoscopic-assisted transanal pull-through (LATP) versus complete transanal pullthrough (CTP) in the surgical management of Hirschsprung's disease. J Pediatr Surg 2016;51:770-4. [CrossRef]

8. Kim AC, Langer JC, Pastor AC, Zhang L, Sloots CE, Hamilton NA, et al. Endorectal pull-through for Hirschsprung's disease-a multicenter, long-term comparison of results: transanal vs transabdominal approach. J Pediatr Surg 2010;45:1213-20. [CrossRef]

9. Stensrud KJ, Emblem R, Bjørnland K. Anal endosonography and bowel function in patients undergoing different types of endorectal pull-through procedures for Hirschsprung disease. J Pediatr Surg 2015;50:1341-6. [CrossRef]

10. Ali KAEE. Transanal Endorectal Pull-through for Hirschsprung's Disease During the First Month of Life. Annals of Pediatric Surgery 2010;6:81-8.

11. Dasgupta R, Langer JC. Evaluation and management of persistent problems after surgery for Hirschsprung disease in a child. J Pediatr Gastroenterol Nutr 2008;46:13-9. [CrossRef]

12. Marty TL, Seo T, Sullivan JJ, Matlak ME, Black RE, Johnson DG. Rectal irrigations for the prevention of postoperative enterocolitis in Hirschsprung's disease. J Pediatr Surg 1995;30:652-4. [CrossRef] 